\title{
Block implementations of the symmetric QR and Jacobi algorithms
}

\section{Report}

\section{Author(s):}

Arbenz, Peter; Oettli, Michael

Publication date:

1992

\section{Permanent link:}

https://doi.org/10.3929/ethz-a-000647001

\section{Rights / license:}

In Copyright - Non-Commercial Use Permitted

\section{Originally published in:}

ETH, Eidgenössische Technische Hochschule Zürich, Departement Informatik, Institut für Wissenschaftliches Rechnen 178 
Eidgenössische

Technische Hochschule

Zürich
Departement Informatik Institut für

Wissenschaftliches Rechnen
Peter Arbenz

Michael Oettli
Block Implementations of the Symmetric QR and Jacobi Algorithms

June 1992 
ETH Zürich

Departement Informatik

Institut für Wissenschaftliches Rechnen

Prof. Dr. W. Gander

Peter Arbenz

Institut für Wissenschaftliches Rechnen

Eidgenössische Technische Hochschule $\mathrm{CH}-8092$ Zürich

e-mail: arbenz@inf.ethz.ch

Michael Oettli

Institut für Wissenschaftliches Rechnen

Eidgenössische Technische Hochschule

$\mathrm{CH}-8092$ Zürich

e-mail: oettli@inf.ethz.ch

This report is also available via anonymous ftp from ftp.inf.ethz.ch as doc/tech-reports/1992/178.ps.

(c) 1992 Departement Informatik, ETH Zürich 


\title{
BLOCK IMPLEMENTATIONS OF THE SYMMETRIC QR AND JACOBI ALGORITHMS
}

\author{
Peter Arbenz and Michael Oettli \\ Institute for Scientific Computing, \\ ETH Zurich, 8092 Zurich, Switzerland
}

\begin{abstract}
A common approach to solve problems in numerical linear algebra efficiently on modern high speed computers is to redesign the classical algorithm, which was originally developed for serial computers. In this paper, we discuss block variants of $\mathrm{QR}$ and Jacobi algorithms for the computation of the complete spectral decomposition of symmetric matrices. We report on numerical tests, which have been performed on a CRAY Y-MP and an ALLIANT FX/80.
\end{abstract}

\section{Introduction}

The QR-algorithm is the most widely used algorithm for finding the eigenvalues and eigenvectors of a symmetric matrix. Subroutines, which implement this algorithm (e.g. TRED2 and TQL2 from EISPACK [21]) perform very well on sequential computers. But, the classical algorithm may be reorganized to exploit the hardware of high performance computers.

On modern supercomputers with vector- and/or parallel-processing capabilities, it is very important to avoid unnecessary memory references, as moving data between different levels of memory (registers, cache, main memory) is slow compared to arithmetic operations on the data. It has turned out, that on vector-processing computers matrix-vector operations can be efficiently implemented (see [10]). On parallel shared memory computers with cache memory only matrix-matrix operations perform optimally (see [13]). These facts has led to the development of new algorithms and redesign of old ones, which are based on these level 2 (matrix-vector) and level 3 (matrixmatrix) operations. This approach is further motivated by the availability of highly optimized BLAS-2 [8] and BLAS-3 [7] library routines on various supercomputers.

A further very popular algorithm for the computation of the complete eigenvalue problem is the algorithm of Jacobi. This algorithm did not make it into EISPACK. But, a very good implementation in ALGOL by Rutishauser can be found in the 'Handbuch' of Wilkinson and Reinsch [18]. We discuss improvements of the original algorithm which concern blocking of Jacobi rotations as well as parallel execution of the blocked rotations.

In this report we examine how these two algorithms may be reorganized to block algorithms and efficiently implemented on high performance computers.

Numerical tests of the algorithms have been made on a CRAY Y-MP and on an ALLIANT $\mathrm{FX} / 80$.

\section{QR Algorithm}

The starting point of this section is a paper by Z. Bai \& J. Demmel [2], which describes a block QR algorithm for real nonsymmetric matrices based on a multishift iteration.

\subsection{The Tridiagonalization}

The symmetric QR algorithm requires the initial reduction of the full matrix to tridiagonal form. This operation is usually performed in the following way. 
Let $A \in \mathbb{R}^{n \times n}$ be the full, symmetric matrix. The algorithm constructs a sequence $A_{0}, A_{1}, \ldots$, $A_{n-2}$ of matrices similar to $A$, such that $A_{n-2}$ has the required tridiagonal form:

$$
\begin{aligned}
& A_{0}=A, \\
& A_{k}=P_{k}^{T} A_{k-1} P_{k}, \quad k=1, \ldots, n-2
\end{aligned}
$$

Each $P_{k}=\left(I-u_{k} u_{k}^{T}\right)$ with $\left\|u_{k}\right\|_{2}=\sqrt{2}$ is a symmetric orthogonal Householder matrix chosen to introduce zeros below the subdiagonal of the $\mathrm{k}$-th column and to the right of the superdiagonal of the $k$-th row of $A_{k-1}$ (see [14] for how to choose the $u_{k}$ ). Further, the similarity transformation can be expressed as a rank-2 update:

$$
A_{k}=P_{k}^{T} A_{k-1} P_{k}=A_{k-1}-u_{k} v_{k}^{T}-v_{k} u_{k}^{T}
$$

where

$$
v_{k}=y_{k}-\frac{1}{2}\left(u_{k}^{T} y_{k}\right) u_{k}, \quad y_{k}=A_{k-1} u_{k}
$$

When the eigenvectors are required too, the Householder matrices have to be accumulated

$$
Q=P_{1} P_{2} \cdots P_{n-2}
$$

To minimize the number of arithmetic operations the accumulation is performed in reversed order.

$$
\begin{aligned}
Q_{n-1} & =I, \\
Q_{k} & =Q_{k+1}-u_{k}\left(u_{k}^{T} Q_{k+1}\right), \quad k=n-2, \ldots, 1
\end{aligned}
$$

This requires only $\frac{4}{3} n^{3}+O\left(n^{2}\right)$ flops. A flop or floating point operation is defined here as a floating point add or multiply (see [14]). The complete reduction algorithm requires $\frac{8}{3} n^{3}+\frac{3}{2} n^{2}+\frac{5}{6} n-29$ flops.

To achieve better memory utilization on parallel-processing computers with hierarchical memory, Dongarra et al. [11] have proposed to aggregate a sequence of $p$ transformations, so that the matrix will be updated by a rank- $2 p$ symmetric matrix. The modified algorithm is as follows: instead of explicitly updating the matrix with the rank two change, only the second column of $A_{2}$, say $a_{2}$ is formed. Then $a_{2}$ is updated by applying the original rank- 2 update:

$$
a_{2}=a_{2}-v_{1}^{(2)} u_{1}-u_{1}^{(2)} v_{1}
$$

where $v_{1}^{(2)}$ and $u_{1}^{(2)}$ denote the second elements of $v_{1}$ and $u_{1}$. From this, $u_{2}$ can be computed and $y_{2}$ and $v_{2}$ are constructed as follows:

$$
\begin{aligned}
& y_{2}=A_{2} u_{2}=\left(A_{1}-u_{1} v_{1}^{T}-v_{1} u_{1}^{T}\right) u_{2} \\
& v_{2}=y_{2}-\frac{1}{2}\left(u_{2}^{T} y_{2}\right) u_{2}
\end{aligned}
$$

Then, $A_{3}$ can be formed as a symmetric rank four update:

$$
\begin{aligned}
A_{3} & =A_{1}-u_{1} v_{1}^{T}-v_{1} u_{1}^{T}-u_{2} v_{2}^{T}-v_{2} u_{2}^{T} \\
& =A_{1}-\left[u_{1} u_{2}\right]\left[v_{1} v_{2}\right]^{T}-\left[v_{1} v_{2}\right]\left[u_{1} u_{2}\right]^{T} .
\end{aligned}
$$

In general we have a rank- $2 p$ update

$$
A_{p+1}=A_{1}-U V^{T}-V U^{T}
$$

where

$$
U=\left[u_{1}, \ldots, u_{p}\right], \quad V=\left[v_{1}, \ldots, v_{p}\right]
$$

Thus $A_{p+1}$ can be formed by a rank- $2 p$ update, which is rich in matrix-matrix operations.

The Householder matrices can be accumulated in a blocked fashion by using the storage efficient WY-representation [19]. The product $P_{1} \cdots P_{p}$ of Householder matrices can be written in the form $I-U T U^{T}$, where $U$ is the matrix of Householder vectors from above and $T$ is a $p \times p$ upper-triangular matrix [19]. Thus, the accumulation is rich in matrix-matrix operations, too:

$$
\begin{aligned}
Q_{m} & =I \\
Q_{k} & =Q_{k+1}-U_{k} T_{k} U_{k}^{T} Q_{k+1}, \quad k=m-1, \ldots, 1, \quad m=n / p
\end{aligned}
$$


The matrix-matrix operations in this block reduction are paid by additional $\left(2 p-\frac{3}{2}\right) n^{2}+O(n)$ flops and extra workspace of size $n \times p$ for $V$ and $p \times p$ for $T$.

We implemented the algorithm in FORTRAN-77 with calls to BLAS primitives of level two and three as subroutine BTRED. An analogous algorithm is implemented in the new library LAPACK [1] as subroutines SSYTRD, DORGQR. Numerical tests are discussed in section 4.

\subsection{Multishift QR Iteration}

Given a symmetric tridiagonal matrix $T \in \mathrm{IR}^{n \times n}$, the $\mathrm{QR}$ algorithm constructs a sequence of similar matrices $T_{i}(i=1,2, \ldots)$, converging to diagonal form. Let us first look at a step of the usual implicit symmetric QR iteration (see [14]):

- Choose a shift $\sigma$. A favourite shift is Wilkinson's (the eigenvalue of T's trailing 2-by-2 principal submatrix closer to the last diagonal element of $T$ ).

- compute first column $m_{1}$ of $M=\left(T_{i}-\sigma I\right)$.

- Determine a Givens rotation $G_{0}$, such that $G_{0}^{T} m_{1}=\mu e_{1}, \mu \in \mathbb{R}$ where $e_{1}$ is the first column of the identity matrix

- Perform similarity transformation with $G_{0}$ on $T_{i}$ :

$$
G_{0}^{T} T_{i} G_{0}=\tilde{T}_{i}=\left[\begin{array}{ccccc}
\times & \times & + & 0 & 0 \\
\times & \times & \times & 0 & 0 \\
+ & \times & \times & \times & 0 \\
0 & 0 & \times & \times & \times \\
0 & 0 & 0 & \times & \times
\end{array}\right]
$$

- Restore tridiagonal form with a sequence of Givens rotations. At any stage, there is only one nonzero entry outside the tridiagonal band.

$$
T_{i+1}=G_{n-2}^{T} \cdots G_{1}^{T} \tilde{T}_{i} G_{1} \cdots G_{n-2}
$$

This QR step requires about $30 n$ flops and additional $6 n^{2}$ flops if the Givens rotations are accumulated.

Now, Bai \& Demmel [2] proposed to choose $k$ shifts simultaneously and to perform $\mathrm{k}$ steps at a time. The benefit of this modification are fewer iterations and more costly steps, which are however rich in matrix-matrix operations. This implicit multishift algorithm may be formulated for the symmetric case as follows:

- Choose $k$ shifts $\sigma_{1}, \ldots, \sigma_{k}$. As [2], we shifted by the $k$ eigenvalues of the $k \times k$ trailing principle submatrix.

- Compute the first column $m_{1}$ of $M$, where $M=\left(T_{i}-\sigma_{1} I\right)\left(T_{i}-\sigma_{2} I\right) \cdots\left(T_{i}-\sigma_{k} I\right)$

- Determine a Householder reflector $P_{0}=I-u_{0} u_{0}^{T}$, such that $P_{0}^{T} m_{1}=\mu e_{1}, \quad \mu \in \mathbb{R}$

- Premultiply and postmultiply $T_{i}$ with $P_{0}: P_{0}^{T} T_{i} P_{0}=\tilde{T}_{i}$

- Restore tridiagonal form with a sequence of Householder transformations.

$$
T_{i+1}=P_{n-2}^{T} \cdots P_{1}^{T} \tilde{T}_{i} P_{1} \cdots P_{n-2}
$$

After pre- and post-multipling $T_{i}$ by $P_{0}, \tilde{T}_{i}$ is a triangular matrix with two symmetric $k \times k$ bulges of nonzero elements outside the tridiagonal band, e.g. for $n=6, k=2$ :

$$
\left[\begin{array}{cccccc}
\times & \times & + & + & 0 & 0 \\
\times & \times & \times & + & 0 & 0 \\
+ & \times & \times & \times & 0 & 0 \\
+ & + & \times & \times & \times & 0 \\
0 & 0 & 0 & \times & \times & \times \\
0 & 0 & 0 & 0 & \times & \times
\end{array}\right]
$$


The bulges of nonzero elements are "chased" down the diagonal in the same way as in the case of a single shift. But now, Householder reflectors may be used instead of Givens rotations. Matrixmatrix operations are introduced, if the Householder reflections are aggregated to chase the bulges $p$ columns at a time. The result is a reduction algorithm, which is basically the same algorithm we used to tridiagonalize the full matrix. In fact, only two adjustments are necessary: the length of the Householder vectors depend now on the number of shifts $k$ and the transformations cannot be accumulated in reversed order anymore.

It must be considered however, that the performance of this reduction, which is the core of the QR iteration, depends on a $k$, that is large enough to exploit the advantages of level 3 operations. But that means additional work of $O\left(n^{2}\right)$ flops per step.

The convergence criterion is the same as in the standard QR algorithm. Negligible small subdiagonal elements are set to zero and the appropriate submatrices are deflated. The algorithm then works on the submatrices. If a submatrix is smaller than some $n_{0}$ depending on $k$, the classical QR algorithm is used to compute its eigenvalues.

We implemented this multishift QR iteration in FORTAN 77 as subroutine BLKQR with calls to BLAS primitives. The $k$ shifts are computed by the EISPACK routine TQL1 and submatrices smaller than $n_{0}$ are handled by TQL2. To simplify the algorithm, we kept the tridiagonal matrix in the $n \times n$ storage place of the initial full matrix. Furthermore, extra workspace of size $n \times p$ for $V$ and $p \times p$ for $T$ is needed for the reduction (see section 2.1). Numerical experiments are reported in section 4 .

\section{The Jacobi Algorithm}

The Jacobi Algorithm for solving the symmetric eigenvalue problem

$$
A \mathbf{x}=\lambda \mathbf{x}
$$

was published by Jacobi in $1846[16]$. He proposed to construct a sequence $\left\{A_{k}\right\}_{k=0}^{\infty}, A_{0}=A$, by

$$
A_{k+1}=J_{k}^{T} A_{k} J_{k}, \quad A_{0}=A,
$$

where the matrices

$$
J_{k}=J\left(p_{k}, q_{k}, \vartheta_{k}\right)=\left(\begin{array}{ccccc}
I_{p_{k}-1} & & & & \\
& c_{k} & & s_{k} & \\
& & I_{q_{k}-p_{k}-1} & & \\
& -s_{k} & & c_{k} & \\
& & & & I_{n-q_{k}}
\end{array}\right)
$$

are so-called plane or Jacobi rotations. Here, $I_{m}$ denotes the identity matrix of order $m, c_{k}=\cos \vartheta_{k}$ and $s_{k}=\sin \vartheta_{k} \cdot \vartheta_{k},\left|\vartheta_{k}\right|<\frac{\pi}{4}$, is chosen such that $a_{p_{k}, q_{k}}^{(k+1)}=0$. In the classical Jacobi algorithm, the pair $\left(p_{k}, q_{k}\right)$ of rotation indices is chosen to zero the off-diagonal element largest in modulus, i.e. $\left|a_{p_{k}, q_{k}}^{(k)}\right|=\max _{p>q}\left|a_{p q}^{(k)}\right|$. Jacobi proved that with this strategy $\left\{A_{k}\right\}$ converges to a diagonal matrix. Let $V_{k}=J_{0} J_{1} \cdots J_{k}$. The convergence of the Jacobi algorithm implies that the orthogonal matrix $V=\lim _{k \rightarrow \infty} V_{k}$ diagonalizes $A$.

While the updates $A_{k+1}=J_{k}^{T} A_{k} J_{k}$ and $V_{k+1}=V_{k} J_{k}$ cost only $12 n$ flops, locating the largest off-diagonal requires $(n-1) n / 2$ comparisons. To avoid this expensive searching, the rotation index pairs can be chosen cyclically, e.g. row-wise, starting with $(p, q)=(1,2)$ and ending with $(p, q)=(n-1, n)$. The most elaborate implementation of this algorithm is by Rutishauser [18].

Rutishauser's Jacobi algorithm is inferior to the QR algorithm with respect to speed on serial computers. QR is about 5 times as fast as Jacobi.

Nevertheless, the Jacobi algorithm has always stayed very popular, on one hand because of its simplicity, on the other hand for its stability and precision [6]. A further reason, why the Jacobi algorithm deserves interest is that it is easily adapted to parallel computation $[3,4,5,12]$. Indeed, if the rotation index pairs are chosen, for instance, along the antidiagonal, in the so-called modulus ordering $[17,20]$, each cycle (sweep) of rotations can be subdivided into $\sim n$ stages of $\sim \frac{n}{2}$ Jacobi 
updates all of which can be executed in parallel. Figure 1 illustrates this selection of rotation index pairs. In this figure, the matrix of rotation index pairs $(p, q)$ are depicted. Rotations which can be executed in parallel are indicated by the same number. A disadvantage of parallel Jacobi

\begin{tabular}{|c|c|c|c|c|c|c|}
\hline$\times$ & 2 & 3 & 4 & 5 & 6 & 7 \\
\hline & $\times$ & 4 & 5 & 6 & 7 & 1 \\
\hline & & $\times$ & 6 & 7 & 1 & 2 \\
\hline & & & $\times$ & 1 & 2 & 3 \\
\hline & & & & $\times$ & 3 & 4 \\
\hline & & & & & $\times$ & 5 \\
\hline & & & & & & $\times$ \\
\hline
\end{tabular}

Figure 1: Rotation index pairs of modulus ordering

algorithms is, that the symmetry of the matrices $A_{k}$ cannot be exploited anymore. The rotations belonging to one stage interfere when they are applied from different sides. Therefore, all rotations of a stage are to be applied as a whole, first on the rows and then on the columns, thus doubling the serial complexity of the parallel algorithm. To avoid this complexity increase, one-sided or implicit Jacobi algorithms have been proposed. We discuss the implementation of Veselić and Hari [23]. One-sided Jacobi algorithms have their origin in the algorithm of Hestenes for the computation of the singular value decomposition of a matrix [15].

Let us assume, that $A=A^{T}$ is positive definite. This is theoretically no restriction as any symmetric matrix can be made positive definite by an appropriate spectral shift. This shift may however worsen the relative accuracy of the computed results [6]. When $A$ is positive definite, its Cholesky decomposition

$$
A=L L^{T}
$$

exists. Set

$$
\tilde{A}=L^{T} L .
$$

$\tilde{A}$ is similar to $A$. (The step from (2) to (3) is actually one step of the symmetric LR algorithm.) We apply the Jacobi algorithm to $\tilde{A}$, constructing a sequence $\{\tilde{A}\}_{k=0}^{\infty}, \tilde{A}_{0}=\tilde{A}$, which converges towards a diagonal matrix $D$. We do not work with the $\tilde{A}_{k}$ explicitly, but with its factor $L_{k}$, where $\tilde{A}_{k}=L_{k}^{T} L_{k}$. Note that $L_{k}$ is not lower triangular for $k>0$.

To determine the rotation angle $\vartheta_{k}$ of $J\left(p_{k}, q_{k}, \vartheta_{k}\right)$ the elements $\tilde{a}_{p_{k} p_{k}}, \tilde{a}_{q_{k} q_{k}}$ and $\tilde{a}_{p_{k} q_{k}}$ must be at hand. While the diagonal elements of $\tilde{A}_{k}$ are typically stored in an auxiliary vector and thus are readily available, the off-diagonal element $\tilde{a}_{p_{k} q_{k}}$ has to be computed as the dot product of the $p_{k}$-th and $q_{k}$-th column of $L_{k}$. The $L_{k}$ are related through

$$
L_{k+1}=L_{k} J_{k}=L_{k} J\left(p_{k}, q_{k}, \vartheta_{k}\right) .
$$

Let $Q_{k}=J_{0} \cdots J_{k-1}$. The Jacobi algorithm constructs a matrix sequence converging towards a diagonal matrix,

$$
\tilde{A}_{k}=L_{k}^{T} L_{k}=Q_{k}^{T} L^{T} L Q_{k} \longrightarrow D, \quad(k \rightarrow \infty) .
$$

So, the matrix sequence $\left\{L_{k}\right\}$ tends to a matrix, say $S=L Q$, where $Q=\lim _{k \rightarrow \infty} Q_{k}$. As the product of orthogonal matrices, $Q$ itself has mutually orthogonal columns. One easily verifies that also $S D^{-\frac{1}{2}}$ is orthogonal. Furthermore,

$$
\begin{aligned}
A S D^{-\frac{1}{2}} & =L L^{T} L Q D^{-\frac{1}{2}} \\
& =L Q D^{-\frac{1}{2}} D D^{-\frac{1}{2}} Q^{T} L^{T} L Q D^{-\frac{1}{2}} \\
& =L Q D^{-\frac{1}{2}} D=S D^{-\frac{1}{2}} D
\end{aligned}
$$

So, the columns of $S D^{-\frac{1}{2}}$ are the normalized eigenvectors of $A$. As the initial Cholesky decomposition of $A$ can be performed in-place, this one-sided Jacobi algorithm needs only the memory for one matrix. The algorithm computes the matrix $S$ from which the eigenvectors of $A$ are obtained 
by normalization of its columns. The norms of the columns of $S$ (in practice of $L_{k}$ for some $k$ ) are A's eigenvalues.

The cost of one iteration step is $8 n$ flops, $2 n$ for the computation of $a_{p_{k} q_{k}}^{(k)}$ and $6 n$ for the update of $L_{k}$. A complete cycle costs $4 n^{3}$ compared with $6 n^{3}$ of the original cyclic Jacobi algorithm. The one-sided algorithm is not only cheaper but also better suited for parallel implementation as the rotations are applied merely from the right. $[9]$.

The initial Cholesky decomposition costs roughly $\frac{1}{3} n^{3}$. It can be parallelized in several ways

Further, it is possible to execute Jacobi's algorithm in blocked form [20, 22]. This means that plane rotations are gathered before they are applied on the full matrix. To be more precise, let

$$
A=\left(\begin{array}{cccc}
A_{11} & A_{12} & \cdots & A_{1 m} \\
A_{21} & A_{22} & \cdots & A_{2 m} \\
\vdots & \vdots & \ddots & \vdots \\
A_{m 1} & A_{m 2} & \cdots & A_{m m}
\end{array}\right) \in \mathbb{R}^{n \times n}
$$

be a $m \times m$ block matrix with blocks $A_{i j} \in \mathbb{R}^{b_{i} \times b_{j}}$ and $b_{1}+\cdots+b_{m}=n$. To perform one block-Jacobi step with the $(r, s)$-block one proceeds as follows:

- Set $\bar{A}=\left(\begin{array}{cc}A_{r r} & A_{s r} \\ A_{r s} & A_{s s}\end{array}\right) \in \mathbb{R}^{\left(b_{r}+b_{s}\right) \times\left(b_{r}+b_{s}\right)}$ and $\bar{V}=I_{b_{r}+b_{s}}$.

- Apply plane rotations $J(p, q, \vartheta)$ with $p=1, \ldots, p_{1}$ and $q=q_{0}, \ldots, b_{p}+b_{q}$ on $A$ and $V$,

$$
\bar{A} \leftarrow J(p, q, \vartheta)^{T} \bar{A} J(p, q, \vartheta), \quad \bar{V} \leftarrow \bar{V} J(p, q, \vartheta) .
$$

We set $p_{1}=b_{p}$ if $r<m$, otherwise $p_{1}=b_{p}+b_{q}$. The value of $q_{0}$ is $b_{p}+1$ if $s>r+1$, otherwise $q_{0}=p+1$.

- Finally, the $r$-th and $s$-th block rows and columns of $A$ and the $r$-th and $s$-th block columns of $V$ are updated,

$$
\begin{aligned}
&\left(\begin{array}{cc}
A_{1 r} & A_{1 s} \\
\vdots & \vdots \\
A_{m 1} & A_{m s}
\end{array}\right) \leftarrow\left(\begin{array}{cc}
A_{1 r} & A_{1 s} \\
\vdots & \vdots \\
A_{m r} & A_{m s}
\end{array}\right) \bar{V} \\
&\left(\begin{array}{ccc}
A_{r 1} & \cdots & A_{s 1} \\
A_{r m} & \cdots & A_{s m}
\end{array}\right) \leftarrow \bar{V}^{T}\left(\begin{array}{ccc}
A_{r 1} & \cdots & A_{s 1} \\
A_{r m} & \cdots & A_{s m}
\end{array}\right) \\
&\left(\begin{array}{cc}
V_{1 r} & V_{1 s} \\
\vdots & \vdots \\
V_{m 1} & V_{m s}
\end{array}\right) \leftarrow\left(\begin{array}{cc}
V_{1 r} & V_{1 s} \\
\vdots & \vdots \\
V_{m r} & V_{m s}
\end{array}\right) \bar{V} .
\end{aligned}
$$

In this way, each off-diagonal element of $A$ is zeroed once per cycle if each off-diagonal block is selected once per block sweep. If the blocks are selected row-wise or in the modulus ordering, the block Jacobi algorithm is equivalent with the (non-blocked) row-cyclic Jacobi algorithm and thus convergent $[17,20]$.

\section{Numerical Tests}

Numerical experiments have been carried out on a CRAY Y-MP/464 at Zurich and on an ALLIANT FX/80 at CERFACS.

All new codes have been written in FORTRAN 77 with 64 bit precision and with calls to appropriate BLAS routines. BLAS-1 subroutines are not used, because both compilers optimized the corresponding FORTRAN loops as well. Unless otherwise stated, the test matrices have been generated randomly for the QR algorithm. As our Jacobi implementation works only with positive definite matrices, those tests have been performed with matrices $A$ with $a_{i j}=n-|i-j|$. 


\subsection{Block Reduction}

The execution times of BTRED, our implementation of the block tridiagonalization algorithm, TRED2 and an additional routine STRED are shown here. TRED2 is the unmodified EISPACK routine. The third subroutine STRED is an implementation of the classical reduction. It differs from TRED2 in that as many calls to BLAS-2 primitives as possible were made.

On the CRAY, all three codes were vectorized by the compiler. Furthermore, the level 2 and level 3 BLAS routines, which are included in the scientific library (SCILIB) run in concurrent mode, if several processors are available. Therefore, the execution times $t$ and the speedups $s$ in table 1 are given for one and four processors respectively. The speedup is defined as the ratio of the execution times between the EISPACK routine and the new routines. The timings for one

\begin{tabular}{|c|c|c|c|c|c|c|c|c|c|c|c|}
\hline \multirow[b]{2}{*}{ Size } & \multirow{2}{*}{$\begin{array}{l}1 \text { proc. } \\
\text { TRED2 } \\
t \text { (sec) }\end{array}$} & \multicolumn{5}{|c|}{1 processor } & \multicolumn{5}{|c|}{4 processors } \\
\hline & & $\begin{array}{l}\text { STRED } \\
t \text { (sec) }\end{array}$ & $s$ & $\begin{array}{l}\text { BTRED } \\
t(\mathrm{sec}) \\
\end{array}$ & $s$ & $(p)$ & $\begin{array}{l}\text { STRED } \\
t \text { (sec) }\end{array}$ & $s$ & $\begin{array}{l}\text { BTRED } \\
t(\mathrm{sec}) \\
\end{array}$ & $s$ & $(p)$ \\
\hline 100 & 0.037 & 0.014 & 2.6 & 0.016 & 2.3 & (4) & 0.024 & 1.5 & 0.024 & 1.5 & $(10)$ \\
\hline 200 & 0.19 & 0.084 & 2.3 & 0.094 & 2.0 & (4) & 0.060 & 3.2 & 0.077 & 2.5 & (10) \\
\hline 300 & 0.53 & 0.27 & 2.0 & 0.29 & 1.8 & (4) & 0.14 & 3.8 & 0.21 & 2.5 & (8) \\
\hline 400 & 1.1 & 0.61 & 1.8 & 0.65 & 1.7 & (10) & 0.27 & 4.1 & 0.40 & 2.8 & (8) \\
\hline 500 & 2.0 & 1.2 & 1.7 & 1.2 & 1.7 & (10) & 0.49 & 4.1 & 0.71 & 2.8 & (8) \\
\hline
\end{tabular}

Table 1: Tridiagonalization on CRAY Y-MP

processor in Table 1 show, that the new routines are more than twice as fast as TRED2 at order 100, but the speedups decrease with increasing orders. Furthermore, STRED is slightly faster than BTRED. The parameter $p$ is that blocksize used in BTRED, for which the best result was obtained. These times on the CRAY confirm, that algorithms with optimal exploitation of matrix-vector operations have an optimal performance on poor vector-processing machines without cache memory and that therefore matrix-matrix operations can only yield small further improvements (compare [10]).

The times of the new routines on four processors show, that BLAS-2 and BLAS-3 primitives are well suited to perform in parallel. They are actually the only part of the algorithm executed in parallel. But BTRED is still inferior to STRED. This shows, that the CRAY Y-MP has a well balanced architecture, i.e. the memory bandwidth is sufficiently high to keep pace with the cpu.

On the ALLIANT, the codes were compiled using the -Ogv -AS options to perform global optimization and vectorization. Optimized BLAS routines are included in the mathematical library, which can be linked to a program using the -lalgebra -lcommon -lmath options. This subroutines are not only vectorized but also parallelized. The times in Table 2 for one processor show,

\begin{tabular}{|c|c|c|c|c|c|c|c|c|c|c|c|}
\hline \multirow[b]{2}{*}{ Size } & \multirow{2}{*}{$\begin{array}{l}1 \text { proc. } \\
\text { TRED2 } \\
t \text { (sec) }\end{array}$} & \multicolumn{5}{|c|}{1 processor } & \multicolumn{5}{|c|}{8 processors } \\
\hline & & $\begin{array}{l}\text { STRED } \\
t \text { (sec) }\end{array}$ & $s$ & $\begin{array}{l}\text { BTRED } \\
t \text { (sec) }\end{array}$ & $s$ & $(p)$ & $\begin{array}{l}\text { STRED } \\
t(\mathrm{sec})\end{array}$ & $s$ & $\begin{array}{l}\text { BTRED } \\
t \text { (sec) }\end{array}$ & $s$ & $(p)$ \\
\hline 100 & 0.867 & 0.805 & 1.08 & 0.878 & 0.99 & (5) & 0.189 & 4.59 & 0.215 & 4.03 & (4) \\
\hline 200 & 6.20 & 5.69 & 1.09 & 5.62 & 1.10 & (10) & 1.20 & 5.17 & 1.20 & 5.17 & (4) \\
\hline 300 & 21.1 & 18.8 & 1.12 & 17.5 & 1.21 & (10) & 4.30 & 4.91 & 3.83 & 5.51 & (5) \\
\hline 400 & 51.8 & 47.9 & 1.08 & 40.0 & 1.30 & (15) & 11.4 & 4.54 & 8.88 & 5.83 & (5) \\
\hline 500 & 103.0 & 95.3 & 1.08 & 76.6 & 1.34 & (15) & 23.4 & 4.40 & 17.1 & 6.02 & (5) \\
\hline
\end{tabular}

Table 2: Tridiagonalization on ALLIANT FX/80

that vectorization causes only minor speedups in both new routines. On eight processors however, concurrency shows a good effect. STRED is more than four times as fast as the EISPACK routine. Further, the block algorithm BTRED is clearly superior to STRED at a problem size greater than 200 . The matrix-matrix-operations are needed to exploit the cache memory (see [13]).

\subsection{Block QR Iteration}

To analyze the performance of our block multishift QR routine BLKQR, we compared it with the unmodified EISPACK routine TQL2. The block size $p$ and the number of shifts $k$ were chosen to 
minimize the running time. Table 3 shows, that BLKQR requires about twice the time of TQL2 on

\begin{tabular}{|c|c|c|c|c|c|c|}
\hline \multirow[b]{2}{*}{ Size } & \multicolumn{2}{|c|}{1 processor } & \multicolumn{4}{|c|}{1 processor } \\
\hline & $\begin{array}{l}\text { TQL2 } \\
t(\sec )\end{array}$ & \#it. & $\begin{array}{l}\text { BLKQR } \\
t(\sec )\end{array}$ & $s$ & \#it. & $(p, k)$ \\
\hline 100 & 0.0890 & 207 & 0.184 & 0.48 & 54 & $(4,8)$ \\
\hline 200 & 0.496 & 399 & 0.918 & 0.54 & 132 & $(4,6)$ \\
\hline 300 & 1.37 & 583 & 2.60 & 0.53 & 196 & $(6,6)$ \\
\hline 400 & 3.00 & 773 & 5.56 & 0.54 & 264 & $(6,6)$ \\
\hline 500 & 5.53 & 960 & 9.51 & 0.58 & 322 & $(6,6)$ \\
\hline
\end{tabular}

Table 3: QR iteration on CRAY Y-MP

one processor of the CRAY. The results on the ALLIANT (see Table 4) are very similar. Minor speedups are only achieved on eight processors. But better speedups up to three are achieved with unmodified TQL2, if some loops are executed in vector-concurrent mode. While TQL2 needs

\begin{tabular}{|c|c|c|c|c|c|c|c|c|c|c|}
\hline \multirow[b]{2}{*}{ Size } & \multicolumn{2}{|c|}{1 processor } & \multicolumn{4}{|c|}{1 processor } & \multicolumn{4}{|c|}{8 processors } \\
\hline & $\begin{array}{l}\text { TQL } 2 \\
t(\sec )\end{array}$ & \#it. & $\begin{array}{l}\text { BLKQR } \\
t(\mathrm{sec}) \\
\end{array}$ & $s$ & \#it. & $(p, k)$ & $\begin{array}{l}\text { BLKQR } \\
t(\mathrm{sec})\end{array}$ & $s$ & \#it. & $(p, k)$ \\
\hline 100 & 1.89 & 213 & 5.29 & 0.36 & 58 & $(4,8)$ & 3.02 & 0.63 & 60 & $(4,8)$ \\
\hline 200 & 13.2 & 411 & 28.0 & 0.47 & 143 & $(4,6)$ & 12.3 & 1.07 & 143 & $(4,6)$ \\
\hline 300 & 44.7 & 609 & 72.0 & 0.62 & 204 & $(6,6)$ & 30.1 & 1.48 & 204 & $(6,6)$ \\
\hline 400 & 106.0 & 797 & 157.0 & 0.68 & 275 & $(6,6)$ & 58.7 & 1.81 & 271 & $(6,6)$ \\
\hline 500 & 205.0 & 994 & 285.0 & 0.72 & 346 & $(6,6)$ & 103.0 & 1.99 & 347 & $(6,6)$ \\
\hline
\end{tabular}

Table 4: QR iteration on ALLIANT FX/80

about $2 n$ iterations, BLKQR needs only $\frac{2}{3} n$ iterations. Bai \& Demmel counted $\frac{1}{4} n$ iterations for their Hessenberg QR algorithm.

Empirically we observed, that deflation happens with the following scheme. Usually after a few iterations a $k \times k$-submatrix is deflated at the tail of the matrix. The larger $k$ is, the fewer iterations are needed. However, more iterations are required for larger $k$ until the first submatrix is deflated and afterward the deflation may happen at any place.

The core of the QR iteration is certainly the optimal choice of the shifts. We did not address this problem and simply used the strategy suggested by Bai \& Demmel.

\subsection{Jacobi}

We investigated three variants of Jacobi's algorithm, all written in Fortran 77.

1. A translation of Rutishauser's ALGOL subprogram [18], which incorporates the row-cyclic Jacobi algorithm. This program is used as a reference.

2. A translation and modification of a Pascal program given by Veselić, which is an implementation of the one-sided algorithm by Veselić and Hari [23]. In contrast to the original Pascal program, the modulus ordering of the rotation index pairs is implemented to make parallel execution of the rotations of one stage possible. The stopping criterion was

$$
\max _{p \neq q} \frac{\left|\tilde{a}_{p q}^{(k)}\right|}{\sqrt{\tilde{a}_{p p}^{(k)} \tilde{a}_{q q}^{(k)}}}<\sqrt{n} \epsilon,
$$

where $\epsilon$ is the machine precision. With this stopping criterion we obtained results with the one-sided Jacobi algorithm which were about as accurate as with Rutishauser's algorithm. If $\left|\tilde{a}_{p q}^{(k)}\right|<\sqrt{n} \epsilon \sqrt{\tilde{a}_{p p}^{(k)} \tilde{a}_{q q}^{(k)}}$ for some $(p, q)$, the corresponding rotation was not performed.

3. A block version of the just given one-sided Jacobi algorithm. The blocks are chosen along the antidiagonal in the modulus ordering. So, the block Jacobi rotations can be performed in 
parallel. Within the blocks the rotations are chosen row-wise. The updating of the temporary matrices $\bar{A}$ and $\bar{V}$ (cf.乌3) was done just as in the adaption of the Rutishauser subroutine in 1. As most of the work of one block step is in forming the off-diagonal block of $\bar{A}$ and in the updating of the block columns of $A$, all rotations of a sweep have been performed in this algorithm. Furthermore, we did not obtain the desired accuracy, when we performed rotations only if $\left|\bar{a}_{i j}\right| / \sqrt{a_{i i} \bar{a}_{j j}}>\sqrt{n} \epsilon, n$ the order of $A$.

\begin{tabular}{|c|c|c|c|c|c|c|c|c|c|c|}
\hline \multirow[b]{2}{*}{ Size } & \multirow{2}{*}{$\begin{array}{c}\text { proc. } \\
\text { VESELIC } \\
t(\mathrm{sec})\end{array}$} & \multicolumn{3}{|c|}{1 processor } & \multicolumn{5}{|c|}{8 processors } & \multirow{2}{*}{$\begin{array}{c}1 \text { proc. } \\
\text { RUT ISH } \\
t \text { (sec) }\end{array}$} \\
\hline & & $\begin{array}{l}\text { VESBLK } \\
t \text { (sec) }\end{array}$ & $s$ & $(p)$ & $\begin{array}{l}\text { VESPAR } \\
t \text { (sec) }\end{array}$ & $s$ & $\begin{array}{l}\text { VESBLK } \\
t(\mathrm{sec})\end{array}$ & $s$ & $(p)$ & \\
\hline 100 & 7.56 & 12.3 & 0.61 & $(12)$ & 2.03 & 3.72 & 3.14 & 2.40 & (8) & 13.0 \\
\hline 200 & 62.2 & 89.1 & 0.70 & (12) & 16.9 & 3.68 & 19.9 & 3.13 & $(8)$ & 106.0 \\
\hline 300 & 225.0 & 262.0 & 0.86 & (16) & 73.1 & 3.08 & 57.4 & 3.92 & (12) & 383.0 \\
\hline 400 & 540.0 & 583.0 & 0.93 & $(20)$ & 194.0 & 2.78 & 129.0 & 4.19 & (16) & 970.0 \\
\hline
\end{tabular}

Table 5: Jacobi on ALLIANT FX/80

Table 5 shows the timings we obtained on the ALLIANT FX/80. The comparison of the times for the Rutishauser RUTISH and modified Veselić implementation VESELIC show the estimated ratio of about $3 / 2$. The ratio is actually a little better which is probably due to the fast Givens rotations used in VESELIC. The speedup we obtained with the parallelization is only about 3.5 on the 8 processor machine. It decreases with increasing matrix order.

On one processor, the blocked implementation VESBLK is somewhat slower than VESELIC, but still considerably faster than RUTISH. On eight processors however, it is superior to the unblocked implementation at matrix sizes exceeding 250 and is more than seven times as fast as Rutishauser's implementation RUTISH at size 400. Table 6 shows the times we measured on the Cray Y-MP.

\begin{tabular}{|c|c|c|c|c|c|c|c|c|c|c|}
\hline \multirow[b]{2}{*}{ Size } & \multirow{2}{*}{$\begin{array}{c}1 \text { proc. } \\
\text { VESELIC } \\
t(\text { sec })\end{array}$} & \multicolumn{3}{|c|}{1 processor } & \multicolumn{5}{|c|}{4 processors } & \multirow{2}{*}{$\begin{array}{c}1 \text { proc. } \\
\text { RUT ISH } \\
t \text { (sec) }\end{array}$} \\
\hline & & $\begin{array}{l}\text { VESBLK } \\
t(\mathrm{sec}) \\
\end{array}$ & $s$ & $(p)$ & $\begin{array}{l}\text { VESPAR } \\
t(\mathrm{sec}) \\
\end{array}$ & $s$ & $\begin{array}{l}\text { VESBLK } \\
t(\mathrm{sec}) \\
\end{array}$ & $s$ & $(p)$ & \\
\hline 100 & 0.55 & 0.80 & 0.69 & (8) & 0.18 & 3.1 & 0.30 & 1.8 & $(8)$ & 0.42 \\
\hline 200 & 3.0 & 4.7 & 0.64 & (8) & 1.0 & 3.0 & 1.7 & 1.8 & (8) & 2.8 \\
\hline 300 & 7.9 & 13.0 & 0.61 & (8) & 2.4 & 3.3 & 5.0 & 1.6 & (8) & 8.8 \\
\hline 400 & 17.0 & 30.0 & 0.57 & (8) & 5.2 & 3.3 & 12.0 & 1.4 & (8) & 20.0 \\
\hline
\end{tabular}

Table 6: Jacobi on CRAY Y-MP

The overhead for the blocked algorithm is to large to be compensated by the BLAS- 3 operations. Interestingly, RUTISH is faster than VESELIC for matrix orders smaller than 200 on this machine.

\subsection{A Comparison of QR and Jacobi Algorithms}

Finally, let us make a comparison between the classical algorithms TRED2/TQL2 and RUTISH and the new block algorithms BTRED/BLKQR and VESBLK to compute the complete spectral decomposition. Positive definite test matrices of the form $Q D Q^{T}$ have been produced, where $D$ is a diagonal matrix with diagonal entries uniformly distributed in $[0, n]$ and $Q$ is the Q-factor of the QR-factorization of a random matrix.

While the classical algorithms ran on one processor of the ALLIANT, the new block algorithms used all eight. The timing results in Table 7 show, that BTRED/BLKQR is fastest. The reason is the high speedup in the tridiagonalization. Although, classical Jacobi is not competitive with the QR algorithm, the blocked parallel Jacobi implementation VESBLK is even faster than TRED2/TQL2.

\section{Conclusions}

The aim of this work was to learn, how new ideas, which were proposed in different papers, effect the performance of the QR and Jacobi algorithms for symmetric matrices on supercomputers. 


\begin{tabular}{|c|l|c|c|c|}
\hline Size & Algorithm & $t(\mathrm{sec})$ & $\max _{i}\left\|T q_{i}-\lambda_{i} q_{i}\right\|_{2}$ & $\max _{i}\left\|\left[q_{1}, \ldots, q_{n}\right]^{T} q_{i}-e_{i}\right\|_{2}$ \\
\hline \hline 100 & TRED2/TQL2 & 2.66 & $0.59093 \mathrm{E}-12$ & $0.62630 \mathrm{E}-14$ \\
& BTRED/BLKQR & 2.97 & $0.16983 \mathrm{E}-11$ & $0.64044 \mathrm{E}-14$ \\
& RUTISH & 14.9 & $0.10199 \mathrm{E}-12$ & $0.26609 \mathrm{E}-14$ \\
& VESBLK & 2.79 & $0.45009 \mathrm{E}-12$ & $0.26723 \mathrm{E}-14$ \\
\hline 200 & TRED2/TQL2 & 19.4 & $0.22968 \mathrm{E}-11$ & $0.10263 \mathrm{E}-13$ \\
& BTRED/BLKQR & 12.7 & $0.29786 \mathrm{E}-11$ & $0.81243 \mathrm{E}-14$ \\
& RUT ISH & 120.0 & $0.30690 \mathrm{E}-12$ & $0.36766 \mathrm{E}-14$ \\
& VESBLK & 17.1 & $0.90176 \mathrm{E}-12$ & $0.31513 \mathrm{E}-14$ \\
\hline 300 & TRED2/TQL2 & 64.5 & $0.32024 \mathrm{E}-11$ & $0.12431 \mathrm{E}-13$ \\
& BTRED/BLKQR & 36.9 & $0.78236 \mathrm{E}-11$ & $0.99906 \mathrm{E}-14$ \\
& RUT ISH & 439.0 & $0.58644 \mathrm{E}-12$ & $0.44042 \mathrm{E}-14$ \\
& VESBLK & 47.1 & $0.17705 \mathrm{E}-11$ & $0.27981 \mathrm{E}-14$ \\
\hline 400 & TRED2/TQL2 & 153.0 & $0.45461 \mathrm{E}-11$ & $0.15090 \mathrm{E}-13$ \\
& BTRED/BLKQR & 71.5 & $0.96399 \mathrm{E}-11$ & $0.11459 \mathrm{E}-13$ \\
& RUT ISH & 1020.0 & $0.10427 \mathrm{E}-11$ & $0.48764 \mathrm{E}-14$ \\
& VESBLK & 110.0 & $0.27734 \mathrm{E}-11$ & $0.25046 \mathrm{E}-14$ \\
\hline
\end{tabular}

Table 7: Total time and accuracy of results on ALLIANT FX/80

It has turned out, that the blocked tridiagonalization is very fast on larger problem sizes. This algorithm combined with a unblocked version for smaller matrices is a very powerful implementation for high performance computers. Actually, the new linear algebra library LAPACK [1] contains such subroutines (SSYTRD, DORGQR).

Our implementation of the multishift QR iteration from Bai \& Demmel is not suited to the symmetric eigenvalue problem. A further reduction of the number of iteration steps would be required to compensate the additional work.

The parallel blocked one-sided Jacobi algorithm turned out to be quite competitive with the QR algorithm on a parallel shared memory computer with cache memory as the ALLIANT FX/80. In situations, where the original matrix is close to diagonal, block Jacobi could be the method of choice.

The CRAY Y-MP has a well balanced architecture, therefore the blocked algorithms are not more efficient than the unblocked ones on this machine.

\section{Acknowledgements}

We would like to thank Prof. K. Veselić for providing us with a Pascal program with his Jacobi implementation and for valuable discussions. We also thank Dr. Ian Duff for making computing time on the Alliant FX-80 of the CERFACS in Toulouse available to us and Michel Dayde for the technical support to use this machine.

\section{References}

[1] E. Anderson, Z. Bai, C. Bischof, J. Demmel, J. Dongarra, J. Du Croz, A. Greenbaum, S. Hammarling, A. McKenney, S. Ostrouchov, and D. Sorensen. LAPACK Users' Guide. Society for Industrial and Applied Mathematics, Philadelphia, PA, 1992.

[2] Z. Bai and J. Demmel. On a block implementation of Hessenberg multishift QR iteration. Int. J. High Speed Comput., 1:97-112, 1989.

[3] M. Berry and A. Sameh. A multiprocessor scheme for the singular value decomposition. In G. Rodrigue, editor, Parallel Processing for Scientific Computing, pages 67-71, Philadelphia, 1989. SIAM.

[4] Ch. Bischof. Computing the singular value decomposition on a distributed system of vector processors. Parallel Computing, 11:171-186, 1989. 
[5] R. P. Brent and F. T. Luk. The solution of singular-value and symmetric eigenvalue problems on multiprocessor arrays. SIAM J. Sci. Statist. Comput., 6:69-84, 1985.

[6] J. Demmel and K. Veselić. Jacobi's method is more accurate than QR. Techn. Rep. 468, Computer Science Department, Courant Institute of Mathematical Sciences, New York University, October 1989.

[7] J. J. Dongarra, J. Du Croz, I. Duff, and S. Hammarling. A proposal for a set of level 3 basic linear algebra subprograms. ACM Trans. Math. Softw., 16:1-28, 1990.

[8] J. J. Dongarra, J. Du Croz, S. Hammarling, and R. J. Hanson. An extended set of fortran basic linear algebra subprograms. ACM Transactions on Mathematical Software, 14:1-17, 1988.

[9] J. J. Dongarra, I. S. Duff, D. C. Sorensen, and H. A. van der Vorst. Solving Linear Systems on Vector and Shared Memory Computers. Society for Industrial and Applied Mathematics, Philadelphia, PA, 1991.

[10] J. J. Dongarra, F. G. Gustavson, and A. Karp. Implementing linear algebra algorithms for dense matrices on a vector pipeline machine. SIAM Review, 26:91-112, 1984.

[11] J. J. Dongarra, S. Hammarling, and D. C. Sorensen. Block reduction of matrices to condensed form for eigenvalue computations. ACM Trans. Math. Softw., 14:215-227, 1989.

[12] P. J. Eberlein and H. Park. Efficient implementation of Jacobi algorithms and jacobi sets on distributed memory architectures. J. Parallel Dist. Comput., 8:358-366, 1990.

[13] K. Gallivan, W. Jalby, and U. Meier. The use of BLAS3 in linear algebra on a parallel processor with a hierarchical memory. SIAM J. Sci. Stat. Comput., 8:1079-1084, 1987.

[14] G. H. Golub and C. F. van Loan. Matrix Computations. The Johns Hopkins University Press, Baltimore, MD, 2nd edition, 1989.

[15] M. Hestenes. Inversion of matrices by biorthogonalization and related results. J. Soc. Indust. Appl. Math., 6:51-90, 1958.

[16] C. G. J. Jacobi. Über ein leichtes Verfahren die in der Theorie der Säcularstörungen vorkommenden Gleichungen numerisch aufzulösen. J. reine angew. Math., 30:51-95, 1846.

[17] F. T. Luk and H. Park. A proof of convergence for two parallel Jacobi SVD algorithms. IEEE Trans. Computers, 38:806-811, 1989.

[18] H. Rutishauser. The Jacobi method for real symmetric matrices. Numer. Math., 9:1-10, 1966. Reprinted in: Linear Algebra, J.H. Wilkinson, C. Reinsch (eds.), pp. 202-211, Springer-Verlag, Berlin, 1971.

[19] R. Schreiber and C. F. Van Loan. A storage efficient WY representation for products of Householder transformations. SIAM J. Sci. Stat. Comput., 10:53-57, 1989.

[20] G. Shroff and R. Schreiber. On the convergence of the cyclic Jacobi method for parallel block orderings. SIAM J. Matrix Anal. Appl., 10:326-346, 1989.

[21] B. T. Smith, J. M. Boyle, J. J. Dongarra, B. S. Garbow, Y. Ikebe, V. C. Klema, and C. B. Moler. Matrix Eigensystem Routines - EISPACK Guide. Lecture Notes in Computer Science 6. Springer-Verlag, Berlin, 2nd edition, 1976.

[22] G. Svenson. A block-Hestenes method for the SVD. Techn. Rep. LiTH-MAT-R-1989-28, Department of Mathematics, Linköping University, September 1989.

[23] K. Veselić and V. Hari. A note on a one-sided Jacobi algorithm. Numer. Math., 56:627-633, 1989. 